\title{
Towards Demand-Oriented Flexible Rerouting of Public Transit Under Uncertainty
}

\author{
Saideep Nannapaneni \\ Wichita State University \\ Wichita, Kansas, USA
}

\author{
Abhishek Dubey \\ Vanderbilt University \\ Nashville, Tennessee
}

\begin{abstract}
This paper proposes a flexible rerouting strategy for the public transit to accommodate the spatio-temporal variation in the travel demand. Transit routes are typically static in nature, i.e., the buses serve well-defined routes; this results in people living in away from the bus routes choose alternate transit modes such as private automotive vehicles resulting in ever-increasing traffic congestion. In the flex-transit mode, we reroute the buses to accommodate high travel demand areas away from the static routes considering its spatio-temporal variation. We perform clustering to identify several flex stops; these are stops not on the static routes, but with high travel demand around them. We divide the bus stops on the static routes into critical and non-critical bus stops; critical bus stops refer to transfer points, where people change bus routes to reach their destinations. In the existing static scheduling process, some slack time is provided at the end of each trip to account for any travel delays. Thus, the additional travel time incurred due to taking flexible routes is constrained to be less than the available slack time. We use the percent increase in travel demand to analyze the effectiveness of the rerouting process. The proposed methodology is demonstrated using real-world travel data for Route 7 operated by the Nashville Metropolitan Transit Authority (MTA).
\end{abstract}

\section{CCS CONCEPTS}

- Applied computing $\rightarrow$ Transportation; • Theory of computation $\rightarrow$ Unsupervised learning and clustering.

\section{KEYWORDS}

Public Transit, Flexible, Rerouting, Demand, Clustering

\section{ACM Reference Format:}

Saideep Nannapaneni and Abhishek Dubey. 2019. Towards Demand-Oriented Flexible Rerouting of Public Transit Under Uncertainty. In 14th International Science of Smart City Operations and Platforms Engineering (SCOPE'19), April 15, 2019, Montreal, QC, Canada. ACM, New York, NY, USA, 6 pages. https://doi.org/10.1145/3313237.3313302

\section{INTRODUCTION}

The transit network in Nashville and other similarly sized cities are challenged with lack of cross-town options as well as low frequency

Permission to make digital or hard copies of all or part of this work for personal or classroom use is granted without fee provided that copies are not made or distributed for profit or commercial advantage and that copies bear this notice and the full citation on the first page. Copyrights for components of this work owned by others than ACM must be honored. Abstracting with credit is permitted. To copy otherwise, or republish, to post on servers or to redistribute to lists, requires prior specific permission and/or a fee. Request permissions from permissions@acm.org.

SCOPE'19, April 15, 2019, Montreal, QC, Canada

(ㄷ) 2019 Association for Computing Machinery.

ACM ISBN 978-1-4503-6703-5/19/04 ..\$15.00

https://doi.org/10.1145/3313237.3313302 of public transit service. Transit routes are generally static in nature, i.e., they operate along pre-determined routes and at a designed frequency; this service is typically referred to as Fixed-Route Transit (FRT) [14]. Such fixed-route systems work well when the people using the transit services are closer to the transit routes; this is typically seen in densely-populated metropolitan cities [11]. Demand Responsive Transit (DRT) [14] systems have become a popular form of public transit in rural and low-demand areas; these transit systems have flexible pick-up and drop-off locations depending on the travel demand. In this paper, we are interested in a specific case of medium-sized cities such as Nashville where addition of fixed routes in the fixed-route transit is not economically possible and demand responsive transit systems are inefficient as the demand in Nashville is higher than the regions (rural areas) where DRT systems are typically used.

The fixed-route strategy may not work in smaller cities where the population are spread across a region. People living in locations not served by the transit network resort to using other forms of transportation such as personal vehicles or ride-sharing services. The problem with private transport is that their efficiency to the travel demand is low, i.e., they carry few people and this results in traffic congestion due to increase in the number of private vehicles, used to meet the travel demand [3].

To avoid such traffic congestion, available public transit needs to be used efficiently, i.e., serve as many people as possible. Moreover, the existing road networks may not be sustainable with the everincreasing travel demand through increased use of private vehicles, as new roads cannot be built at the same rate of increase in travel demand. Efficient and reliable use of public transit resources can help streamline the traffic flow process. Furthermore, they are safer compared to the use of automotive vehicles in terms of the number of accidents per passenger mile [9] and also the use of public transit services makes the people more healthy as it requires for them to walk/bike to the nearest bus stop [5].

However, typically transit services, are routed through fixed predetermined travel stops and pre-determined schedule, while it is known that the travel demand shows both spatial and temporal variability, where the number of people using the transit services appear with the time of day and the geographic locations. In our previous work [16], we showed that a transit schedule that varies with seasons is more efficient compared to a static schedule, however, as discussed in that paper even seasonal variations are not optimal and we need a more flexible public transit system.

Flexible transportation systems (FTS) [12] can be considered as a hybrid of FRT and DRT, and are increasingly becoming popular in regions where DRT and FRT are inefficient. Several forms of FTS exist varying from near-FRT to near-DRT systems such as Route Deviation, Point Deviation, Demand Responsive Connector, Request 
Stops, Flexible Route Segments and Zone Routes [12]. Depending on the region and the nature of travel demand, an appropriate FTS is used. In this paper, we utilize the advantages of FTS, particularly the Route Deviation service, to accommodate the spatial and temporal variation of travel demand in medium-size cities such as Nashville. Route Deviation refers to the FTS, where a bus deviates from its fixed route, to accommodate any passenger or para-transit requests to pick-up and drop-off at requested locations.

Challenges: Previous literature $[10,13,14]$ have studied Route Deviation-based FTS; however, the bus stops made in the deviated routes are determined based on the passenger requests and the local transit authority. Such human-involved decision-making regarding the route deviations and flex stops may not be feasible in the presence of large number of passenger requests as the number of possible route deviations increases rapidly. In this paper, we propose an efficient and automated approach based on clustering and discrete optimization to identify the flex stops and the best route deviation to maximize the accessibility of the public transit.

Research Contributions: The overall contributions made in this paper are: (1) Identification of flex bus stops through densitybased clustering, and (2) Computation of the best rerouting strategy through a discrete optimization to serve as many people as possible and thus reduce travel congestion, while still serving fixed route stops identified as critical.

Paper Organization: Section 2 provides the necessary definitions, discusses the issues that need to be addressed and illustrates them using Route 7 operated by the Nashville Metropolitan Authority (MTA). Section 3 provides the assumptions and discusses the proposed solution methodology for rerouting public transit under spatial and temporal variations of travel demand. Implementation of the solution for Route 7 operated by Nashville MTA is detailed in Section 4. Concluding remarks and future work follow in Section 5.

\section{PRELIMINARIES}

\subsection{Definitions}

Scheduled Route: A bus route between start and end points, with pre-determined stops, as scheduled by a local transit authority.

Critical bus stops: Critical bus stops refer to the bus stops that can not be ignored, i.e., buses have to halt at these bus stops. In this paper, the following bus stops are treated as critical bus stops bus stops: (1) Bus stops with historical high passenger demand, and (2) Transfer points, i.e., bus junctions where people change buses to reach their destinations.

Non-critical bus stops: As the name suggests, these bus stops can be ignored by the buses when there is no potential boarding or getting off activity at the bus stops. This activity will be derived from mobile applications and on-call kiosks at the transit-stops [7].

Flex Route: Flex route refers to a deviated route from the original scheduled route in order to be accessible to more people. Flex route are beneficial as they help eliminate the traffic congestion caused when the people on the flex route take cabs or ride sharing services to reach nearby bus stops or their final destinations.

Flex Stops: Flex-stops are new dynamic stops on a flex route where people can gather and board the bus. This is an on-demand feature (but not real-time, see the assumptions below in Section 2.2) and works through deviations around the non-critical stops.
Trip: A trip is defined as a bus journey from the start point to the end point of a scheduled route.

Trip segment: A trip segment is a portion of a trip, which consists of fewer transit stops compared to the overall trip.

Trip Block: A trip block represents a sequence of trips between the start and end bus stops by the same bus.

\subsection{Assumptions}

(1) The process of rerouting from the scheduled route does not occur in real time. The rerouting is done ahead of time, such as the previous night of any day. This is essential to inform the public via phones and kiosks about the changed routes.

(2) We assume the availability of slack time at the end point of a trip.

(3) The departure time at the start point does not change while the arrival time at the end can change but any delay should be less than available slack time. The start and end points are also considered critical stops.

(4) Any extra costs (eg. fuel) that incur due to taking flex routes are ignored.

(5) We show flexible rerouting of a single bus. In future, the proposed methodology will be extended to simultaneous rerouting of several buses.

(6) We assume the data regarding the spatial variation of the travel demand is available. Determination of such spatial distribution from historical data, mobile application data and census data is not demonstrated in this paper.

(7) We consider only pick-up of passengers and not their drop-off.

\section{PROPOSED REROUTING FRAMEWORK}

The proposed methodology for flexible rerouting is performed in three steps: (1) Discretization of real time, (2) Estimation of spatial distribution of travel demand, (3) Identification of Flex Stops, and (4) Rerouting to accommodate flex and critical stops. All the steps are detailed below.

\subsection{Discretization of real time}

The temporal variation is considered by discretizing real time into several time intervals. The length of time intervals is chosen depending on the spatial and temporal variation of travel demand. We propose two ways for the determination of the length of a time interval: (1) Time interval, which equals the time taken for one trip, and (2) Time interval, which represents the time taken to cover a set of trips. For example, time taken to operate two or three trips. In the first option, travel demand is estimated for each trip and accordingly the rerouting is performed. In the second option, an aggregated travel demand over all the trips is considered within the time interval, and rerouting is performed for all the trips simultaneously. If the travel demand for a given trip is not consistent and is associated with large variation, then the rerouting results may not be accurate. An example for a variation in the travel demand is when some of the trip's travel demand is spilled over to the adjacent trips. One reason could be when people are transferring buses; a delay in the incoming bus can result in a person missing the bus and thus reducing the travel demand. Thus, the second option can be 
comparatively robust under variations of travel demand across adjacent trips, when compared to the first option. This paper considers the second option and equal time intervals of 1 hour.

\subsection{Estimation of travel demand}

As mentioned in Section 1, the spatial distribution of the travel demand is available for further analysis. One of the possible approaches for the determination of the spatial distribution of the travel demand is by using the historical Transit-HUB [15] application data, and transaction data from the local transit authorities. The Transit-HUB application is similar to the Google Maps application but also provides real-time delay information for an accurate trip planning. We can use the historical travel patterns; which shows that the travel demand in a region is not a deterministic quantity but stochastic in nature. Therefore, the travel demand within a particular region can be represented through a probability distribution. Such probability distributions at several spatial regions may be used to estimate uncertainty in the spatial distribution of the travel demand.

\subsection{Identification of Flex Stops}

Given the spatial distribution of the travel demand, we identify the spatial locations with high travel demand around them and flex stops are introduced near them. In this paper, the flex stops are identified by performing clustering over the spatial distribution of travel demand. We use the DBSCAN, a density-based clustering algorithm [4]. The DBSCAN algorithm requires two inputs: the maximum distance between the points $(\epsilon)$ and the minimum number of points, minPoints, required to form a cluster. A brief introduction to the DBSCAN algorithm is provided below.

Given a set of data points, we start by choosing an arbitrary data point. We identify the number of data points that are within the $\epsilon$ distance from the chosen point. If the number of data points (including itself) is less than the minPoints, then that point is consider as an outlier. If the number of points within the $\epsilon$ distance is greater than the minPoints, all such points including the original point form an initial cluster. The initial cluster is then recursively expanded in size by checking if any points inside it have atleast minPoints around them within the $\epsilon$ distance. If the cluster cannot be expanded any further, the same process is repeated choosing another point, which is not considered before. Several distance measures are available to compute the distance between two data points, but the most commonly used measure is the Euclidean distance [18]. The output from the DBSCAN are the density-based clusters; the centers of the clusters are calculated and treated as the flex stops. We perform density-based clustering for a given spatial demand within a given time interval.

\subsection{Rerouting to serve flex stops}

Figure 1 shows the scheduled route with critical and non-critical bus stops along with possible flex routes. The flex stops indicated in Figure 1 refer to the cluster centers obtained from the clustering of spatial travel demand in Section 3.3. The notation for several variables used in the problem formulation and their description are provided in Table 1.

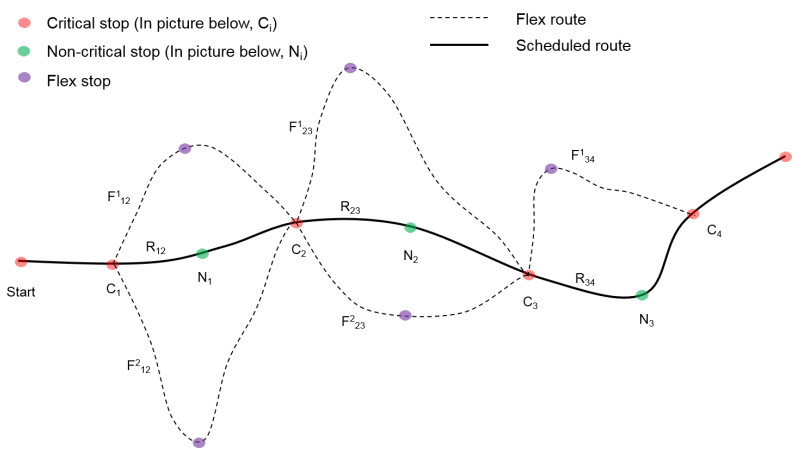

Figure 1: Conceptual scheduled route and possible flex route options by substituting non-critical bus stops for flex stops

Table 1: Variable Notation and its description

\begin{tabular}{|l|l|}
\hline Variable & Description \\
\hline$R_{i k}$ & $\begin{array}{l}\text { Scheduled route between consecutive critical stops } C_{i} \text { and } C_{k}, \\
k=i+1 .\end{array}$ \\
\hline$T\left(C_{i}\right)$ & Departure time at a critical stop $C_{i}$. \\
\hline$T^{s}\left(C_{i}\right)$ & Scheduled departure time at a critical stop $C_{i}$. \\
\hline$F_{i k}^{j}$ & $\begin{array}{l}\text { Flex route segment for } R_{i k} \text {. Superscript } j \text { represents index of flex } \\
\text { route }\end{array}$ \\
\hline$P_{i k}$ & Represent either a segment of scheduled route or a flex route \\
\hline$D\left(P_{i k}\right)$ & Number of people served on the segment $P_{i k}$ \\
\hline$C\left(P_{i k}\right)$ & Additional congestion caused when a segment $P_{i k}$ is not catered \\
\hline$t\left(P_{i k}\right)$ & Actual time taken to travel a segment $P_{i k}$ \\
\hline$t^{s}\left(P_{i k}\right)$ & Time taken to travel a segment $P_{i k}$ according to the schedule \\
\hline$d\left(P_{i k}\right)$ & $\begin{array}{l}\text { Delay caused due to a route } P_{i k} \text { compared against the schedule } \\
\text { route } R_{i k}, d\left(P_{i k}\right)=t\left(P_{i k}\right)-t^{s}\left(R_{i k}\right)\end{array}$ \\
\hline$t(s)$ & Slack time available at the end point \\
\hline$\psi$ & Radius of travel demand that a bus stop can cover \\
\hline$G_{1}\left(D\left(P_{i k}\right)\right)$ & $\begin{array}{l}\text { Loss Function associated with the people not serviced by the public } \\
\text { transit in segment } P_{i k}\end{array}$ \\
\hline$G_{2}\left(d\left(P_{i k}\right)\right)$ & Loss function associated with travel time delay in a segment $P_{i k}$ \\
\hline$N_{c}$ & Number of critical stops, including the start and end points \\
\hline$\eta_{i}$ & Threshold departure time delay value at the $i^{t h}$ critical stop \\
\hline$p_{i}$ & $\begin{array}{l}\text { Threshold probability regarding the departure time delay at the } \\
i^{t h} \text { critical stop }\end{array}$ \\
\hline
\end{tabular}

Given the notation for several variables, the optimization problem for choosing the optimal flex route can be derived by solving the following optimization problem.

$$
\begin{gathered}
\operatorname{Min} \sum_{i=1}^{N_{c}-1} E\left[w_{1} \times G_{2}\left(d\left(P_{i k}\right)\right)-\left(1-w_{1}\right) \times G_{1}\left(D\left(P_{i k}\right)\right)\right] \\
\text { subject to } \operatorname{Pr}\left(\sum_{i=1}^{N_{c}-1} d\left(P_{i k}\right) \geq t_{s}\right)<\gamma \\
T\left(C_{i}\right)>T\left(C_{j}\right), \quad \forall i>j \\
\operatorname{Pr}\left(T\left(C_{i}\right)-T^{s}\left(C_{i}\right) \geq \eta_{i}\right)<p_{i}, \quad i=1 \cdots N_{c}
\end{gathered}
$$

The overall minimization function is a weighted combination of two loss functions: (1) people served, and (2) time delay in taking a scheduled route or a flex route. The weights for the combination of the two objective functions $\left(w_{1}\right)$ and $\left(1-w_{1}\right)$, and the cost functions associated with the number of people not served and time delay may be obtained from the local transit authority. The weights $\left(w_{1}\right)$ and $\left(1-w_{1}\right)$ represent the importance of the two 
objective functions; higher $w_{1}$ represents more emphasis on minimizing the travel delays while a lower $w_{1}$ value represents places more emphasis on serving people. Since the travel demand and time delay are uncertain, we optimize their expectation values. The optimization is carried out under three constraints: (1) probabilistic total delay constraint, (2) Ordering of the critical stops, and (3) Time delay at the critical bus stops on the scheduled route. $\gamma$ refers to the threshold probability value $(0 \leq \gamma \leq 1)$, which can be provided by the local transit authorities. Lower values of $\gamma$ correspond to tighter constraints while higher values of $\gamma$ result in loose constraints. The second constraint stipulates that the sequence of critical bus stops remain the same as in the original schedule. The third constraint specifies that the new departure time at the critical stops should be within desired delays of the scheduled departure time. $\eta_{i}, i=1,2,3 \cdots N_{c}$ represents the affordable departure delay at a critical stop and $p_{i}, i=1,2,3 \cdots N_{c}$ represents threshold probability, i.e., the probability that the actual departure time at a critical stop is greater than the scheduled departure time. Larger time gaps between the scheduled and flex-route times can result in disruptions of people's travel plans.

To compute the number of people served, we define a parameter $\psi$, which represents a radius around a bus stop (critical, non-critical or flex). If a person lies within the $\psi$ distance, then the person is assumed to be served by the bus. Given a particular route (either scheduled or fixed), the number of people that are served can be computed, using the estimated spatial distribution of travel demand, the flex stops and $\psi$. An estimate of the time delay along a scheduled or a flex route is obtained using the Google Maps or equivalent applications. The delay prediction is usually a point-value but in reality, the actual delay may not match the predicted delay. Therefore, we assume that the delay prediction is quantified through a probability distribution. We use a lognormal distribution as it has been previously used for modeling the travel time distribution [8]. The optimization is subject to a probabilistic constraint with respect to the overall time delay, which is the aggregation of time delays over all the individual segments. The optimization analysis helps decide whether a flex route need to be taken as against a segment the scheduled route. In case there are multiple possible flex routes, the flex route that minimizes the overall loss function is selected.

Effectiveness of the Rerouting process: The next step after performing the rerouting analysis is to decide if the new flex route needs to be operated in place of the scheduled route. Rerouting a scheduled route involves notifying people regarding the new flex route. Frequent changes to the scheduled routes can cause discomfort to regular passengers; therefore, we use the percent increase in travel demand served on a flex route to quantify the effectiveness of the rerouting process. If $D_{F}$ and $D_{S}$ represent the total travel demand served on the flex and scheduled routes, then the percent increase (denoted as $P_{D}$ ) in travel demand served on the flex route is calculated as $P_{D}=\frac{D_{F}-D_{S}}{D_{S}} \times 100 \%$.

We then define a threshold percentage $\Delta$, and if $P_{D}>\Delta$, then the new flex route is operated else the scheduled route is operated. The threshold $\Delta$ can be determined by the local transit authorities. In this way, we determine if a flex or scheduled route needs to be operated. The optimization formulation described above considers the uncertainty in travel demand and travel time, and performs
Table 2: Departure delay parameters at the critical stops

\begin{tabular}{|l|l|l|}
\hline Critical stop ID & $\eta_{i}(\min )$ & $p_{i}$ \\
\hline 1 & 0.5 & 0.01 \\
\hline 2 & 2 & 0.01 \\
\hline 3 & 3 & 0.01 \\
\hline 4 & 6 & 0.01 \\
\hline
\end{tabular}

rerouting under a probabilistic constraint in the overall travel delay. A common approach for solving such stochastic optimization problems is through Monte Carlo Sampling, where for each possible flex route option, multiple realizations of the travel demand and travel time are generated to evaluate the probabilistic objective and constraint functions. For the sake of demonstration, we present the implementation and results below considering only one realization of travel time and travel demand.

\section{IMPLEMENTATION AND RESULTS}

Problem Parameters: For simplicity, we do not consider any losses due to additional travel delay $\left(G_{2}\left(d\left(P_{i k}\right)\right)\right.$ in Table 1). Not considering the loss due to additional travel delay signifies that we are completely committed to accommodating as much travel demand as possible under the slack time constraint.

We considered real transaction data occurring on several trips taken on Route 7 between 9 am and 10 am, on every Monday in October 2016. The $\gamma$ parameter, which represents the delay threshold is set to 1 . This represents a stringent requirement that the additional travel time should always be less than the available slack time, which is assumed to be equal to $7 \mathrm{~min}$. In this example, we have four critical stops (including start and end stops), as shown in Fig. 2. The departure delay parameters $\left(\eta_{i}\right.$ and $p_{i}, i=1$ to 4$)$ are given in Table 2 . The parameter, $\Delta$, that represents the threshold increase in travel demand is assumed to be $10 \%$.

Estimating Travel Demand: We use a combination of transaction data from Route 7 and other nearby bus routes ( 21 bus routes in total) also going to Music City Central available from the Nashville MTA. For illustration, we assumed that $50 \%$ of travel demand to the Music City Central use private transit modes (driving their own vehicles or ride-sharing services), while the remaining use public transit. Note that these numbers represent the proportions of travel demand around Route 7, and do not represent the entire travel characteristics in Nashville. The steps for simulating the travel demand used for rerouting analysis is given below:

(1) Data pre-processing: The original transaction dataset obtained from Nashville MTA had timestamp, vehicle, location and route, but lacks some necessary information, such as direction, trip, and stop. To extend the original transaction data and obtain complete information of each transaction, we pre-processed the dataset by linking the time stamp with static schedule of Route 7. This helped us obtain detailed information of every trip on Route 7.

(2) Data filtering: Transaction data is considered as valid travel demand if the transaction happens within 1 mile from the route and the transaction time is within a period that is between 30 minutes before the time window starts and 30 minutes after the time window ends. MongoDB [6] was used to store the transaction data as geospatial objects, which enabled quick geographical query. 


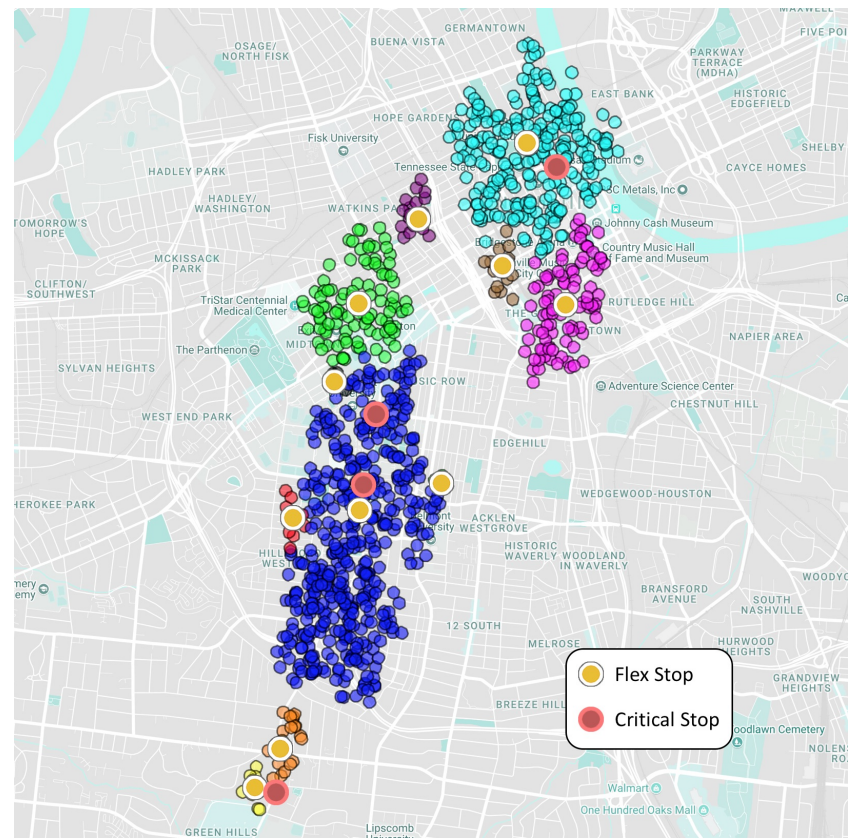

Figure 2: Clusters of the travel demand obtained from DBSCAN clustering, their cluster centers(flex stops), and critical stops.

(3) Demand estimation: In order to better estimate the overall travel demand, the original transaction data that happened near bus stops is used to generate isotropic Gaussian blobs. We doubled the transaction data to get the total travel demand following the above mentioned assumption that the transaction data represents half of the total travel demand, and then distributed those points to nearby areas following Gaussian distribution.

Identification of Flex Stops: After obtaining the overall travel demand, we standardized the travel demand data by removing the mean and scaling to unit variance. Density-based clustering using DBSCAN was then performed to identify flex stops. The different clusters, their centers (flex stops) and critical bus stops (obtained from original Route 7 schedule) are shown in Figure 2. As discussed in Section 3.3, the DBSCAN algorithm has two main parameters - $\epsilon$ and minPoints. Based on the generated travel demand distribution, we experimentally set the parameters ( $\epsilon$ and minPoints) to 0.12 and 10 respectively. As some flex stops fell in locations not reachable by the public transit, the closest locations on the major roads were considered as flex stops.

Determination of the Best Flex Routes: Given the locations of flex and known critical stops, we obtain the best possible flex route by solving the discrete optimization problem in Section 3.4.

Generating possible flex routes: Several valid flex routes, i.e., the routes that satisfy the constraints are generated. The generated flex routes contain all the critical bus stops from the original route and a subset of identified flex routes enabled by the slack time constraint. Using scheduling data from Nashville MTA and Open-Trip Planner (OTP), we created a travel time matrix for all critical and flex stops

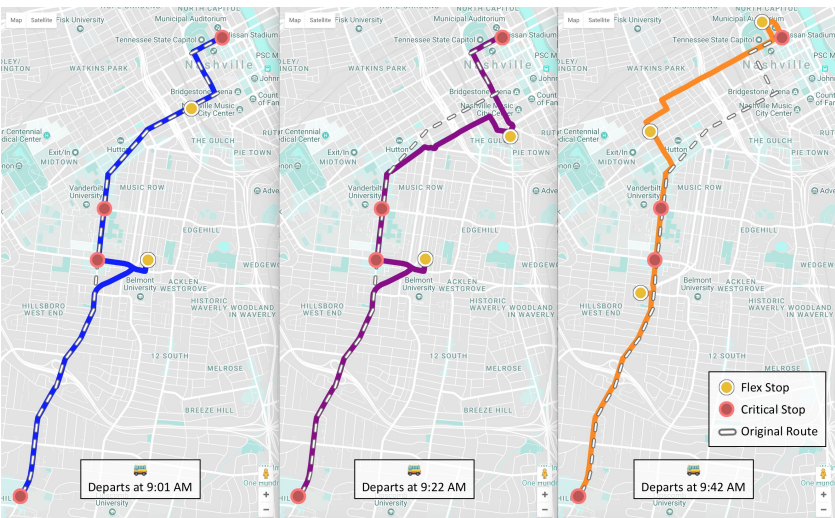

Figure 3: Optimal flex routes showing the critical and flex stops, along with scheduled route, for three trips on Route 7 departing at 9:01 AM, 9:22 AM and 9:42 AM.

and estimate the total travel time for each possible stop sequence combination.

Selecting the routes with most demand coverage: For a bus route in a selected time window, there are usually more than one bus trips that depart in the same direction. In our example of Route 7, there are three trips from Hillsboro High School to Music City Central between the considered time window ( $9 \mathrm{am}$ and $10 \mathrm{am}$ ). Since the choice of flex routes for one route will affect the simulated demand coverage of its next trip, we perform rerouting of all three trips individually but evaluate their new routes altogether to maximize the travel demand served by the buses. In order to decide which bus trip should be changed from the original route to a flex route, we use the Genetic Algorithm (GA) framework. In this context, an individual/chromosome (these two words will be used interchangeably), which represent a sample in the GA, is a solution of how flex routes are scheduled for the bus trips within a time window, and a population is a set of solutions. Each chromosome is an array of integers, of which each integer is the index of a selected flex route for a trip. Particularly, the index 0 is reserved for the original route, which means a trip will not be changed to any flex route. For example, $[0,10,4]$ represents that three trips in the time window: (1) the $1^{\text {st }}$ trip still follow the original route, (2) the $2^{\text {nd }}$ trip uses the $10^{\text {th }}$ flex route, (3) the $3^{r d}$ trip uses the $4^{t h}$ flex route.

Details of the genetic algorithm (GA) runs are given below. We chose the initial population size for GA algorithm to be 50 . The fitness function is travel demand coverage of each individual solution. The parameter $\psi$, which is the radius of travel demand that a bus stop can cover, determines the number of people served by the bus at each stop is assumed to be equal to 0.25 mile. We use uniform crossover [17] to exchange the flex route choices between individuals. The crossover rate is assumed as $40 \%$. In each iteration, $20 \%$ of route choices in a population will be randomly changed to use another path. We set two conditions for termination of the GA algorithm: (1) if the number of iterations is larger than 100, (2) if the best demand coverage has not increased in the most recent three iterations.

Simulation Results: As mentioned above, there are three trips within the considered time interval that depart at 9:01 AM, 9:22 


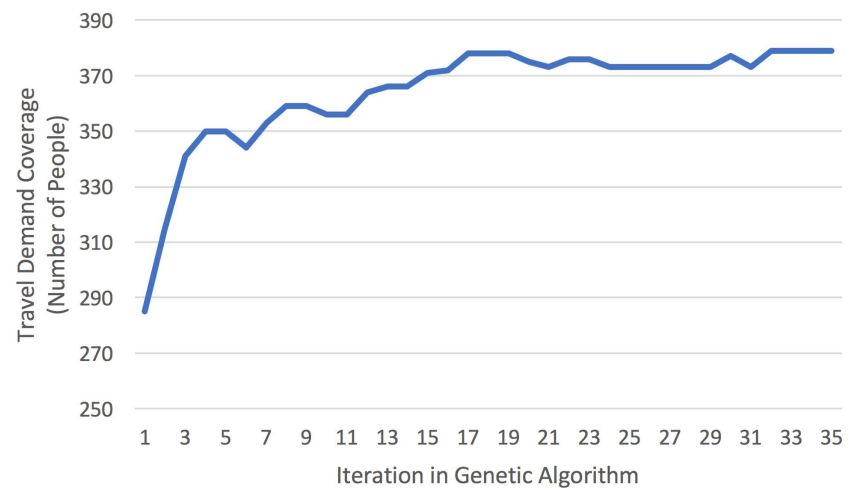

Figure 4: Travel demand coverage with iteration of the optimization process

AM and 9:42 AM [1]. We first estimate the travel demand around the route, identify flex stops for each demand cluster, and finally determine the best flex routes running the genetic algorithm. The best flex route choices for these trips are illustrated in Figure 3 . Figure 4 shows how the travel demand coverage changes in each iteration when the genetic algorithm runs. The original routes only cover travel demand of 285 people, and our flex route solution gets an coverage of 379 people, which is a $32.98 \%$ improvement.

\section{CONCLUSION AND FUTURE WORK}

This paper proposed a flexible rerouting framework for the public transit to better serve the spatial and temporal varying travel demand. The temporal changes in the travel demand are analyzed by discretizing the real time into uniform time intervals and within each interval, rerouting considering spatial variations of travel demand is considered. The bus stops on the scheduled routes are categorized are critical and non-critical stops, the latter are the stops with traditionally low demand and can be ignored. Instead, the buses are rerouted to high-demand areas to accommodate more people and thus, reduce the congestion on the roads, caused when those people resort to private transit modes due to inaccessibility to the public transit. Rerouting requires identifying the geographic locations with high travel demand and this is achieved through clustering. Density-based clustering using DBSCAN algorithm is used to estimate high travel demand areas and the cluster centers are identified as flex stops. If clusters with high densities are identified away from the scheduled transit routes, then the buses are rerouted through the flex stops. Rerouting of public transit incurs additional travel time, which needs to be less than the available slack time at the end of the scheduled route.

The proposed methods were demonstrated for Route 7 operated by the Nashville Metropolitan Transit Authority (MTA). The travel demand was estimated using the real-world transaction data obtained from the Nashville MTA. This work considered rerouting of a single bus under varying travel demand. Future work should consider multiple buses operating in the same geographic region, and perform a multi-agent flexible rerouting analysis. We should also investigate game theory approaches such as providing incentives for taking public transit [2] towards reaching sustainability in traffic flow networks. This paper used percent increase in travel demand as a metric for quantifying the effectiveness of rerouting process.
We will also use the total miles walked by all people and also number of miles per person as a metric for analyzing the effectiveness of rerouting process.

\section{ACKNOWLEDGEMENT}

The research reported in this paper was supported by funds from the National Science Foundation under the award number CNS1528799 and CNS-1647015, and the Vanderbilt Initiative on Smart City Operations and Research, a trans-institutional initiative funded by the Vanderbilt University. The support is gratefully acknowledged. The authors also acknowledge the help from our partners at Nashville Metro Authority. Any findings and conclusions made in this paper are those of the authors and do not represent the views of the funding agencies or the Nashville MTA. The authors would also acknowledge Dr. Fangzhou Sun for his assistance regarding data processing and optimization analysis.

\section{REFERENCES}

[1] [n. d.]. Route 7 of Nashville MTA. http://www.nashvillemta.org/maps/route7.pdf. Accessed: 2019-02-13.

[2] [n. d.]. The Rideshare Incentive Program. http://www.yolocommute.net/ public-transit-incentive-program/. Accessed: 2019-02-13.

[3] Md Aftabuzzaman, Graham Currie, and Majid Sarvi. 2010. Evaluating the congestion relief impacts of public transport in monetary terms. Fournal of Public Transportation 13, 1 (2010), 1.

[4] Martin Ester, Hans-Peter Kriegel, Jörg Sander, Xiaowei Xu, et al. 1996. A densitybased algorithm for discovering clusters in large spatial databases with noise.. In Kdd, Vol. 96. 226-231.

[5] Amy L Freeland, Shailendra N Banerjee, Andrew L Dannenberg, and Arthur M Wendel. 2013. Walking associated with public transit: moving toward increased physical activity in the United States. American journal of public health 103, 3 (2013), 536-542.

[6] Yunhua Gu, Shu Shen, Jin Wang, and Jeong-Uk Kim. 2015. Application of NoSQL database MongoDB. In Consumer Electronics-Taiwan (ICCE-TW), 2015 IEEE International Conference on. IEEE, 158-159.

[7] Taewook Heo, Kwangsoo Kim, Hyunhak Kim, Changwon Lee, Jae Hong Ryu, Youn Taik Leem, Jong Arm Jun, Chulsik Pyo, Seung-Mok Yoo, and JeongGil Ko. 2014. Escaping from ancient Rome! Applications and challenges for designing smart cities. Transactions on Emerging Telecommunications Technologies 25, 1 (2014), 109-119.

[8] Timothy Hunter, Ryan Herring, Pieter Abbeel, and Alexandre Bayen. 2009. Path and travel time inference from GPS probe vehicle data. NIPS Analyzing Networks and Learning with Graphs 12, 1 (2009).

[9] Todd Litman. 2015. Evaluating public transit benefits and costs. Victoria Transport Policy Institute.

[10] Xiaolin Lu, Jie Yu, Xianfeng Yang, Shuliang Pan, and Nan Zou. 2015. Flexible feeder transit route design to enhance service accessibility in urban area. fournal of Advanced Transportation (2015).

[11] Seyed Mohammad Nourbakhsh and Yanfeng Ouyang. 2012. A structured flexible transit system for low demand areas. Transportation Research Part B: Methodological 46, 1 (2012), 204-216.

[12] John F Potts, Maxine A Marshall, Emmett C Crockett, and Joel Washington. 2010. A guide for planning and operating flexible public transportation services. Number Project B-35.

[13] Feng Qiu, Wenquan Li, and Chengchuan An. 2014. A Google Maps-based flexroute transit scheduling system. In CICTP 2014: Safe, Smart, and Sustainable Multimodal Transportation Systems. 247-257.

[14] Luca Quadrifoglio. 2005. A hybrid fixed and flexible transportation service: description, viability, formulation, optimization and heuristic. Technical Report.

[15] Shashank Shekhar, Fangzhou Sun, Abhishek Dubey, Aniruddha Gokhale, Himanshu Neema, Martin Lehofer, and Dan Freudberg. [n. d.]. Transit hub. Internet of Things and Data Analytics Handbook ([n. d.]), 597-612.

[16] F. Sun, C. Samal, J. White, and A. Dubey. 2017. Unsupervised Mechanisms for Optimizing On-Time Performance of Fixed Schedule Transit Vehicles. In 2017 IEEE International Conference on Smart Computing (SMARTCOMP). 1-8. https://doi.org/10.1109/SMARTCOMP.2017.7947057

[17] Gilbert Syswerda. 1989. Uniform crossover in genetic algorithms. (1989).

[18] Rui Xu and Donald Wunsch. 2005. Survey of clustering algorithms. IEEE Transactions on neural networks 16, 3 (2005), 645-678. 